\title{
Galactic Cosmic-Ray Anisotropy During Forbush Decreases: Evidence for Diffusive Barriers and Large-Scale Flow
}

\author{
G.D. Ihongo ${ }^{* a, b}$, D. Ruffolo ${ }^{a}$, A. Sáiz ${ }^{a}$, U. Tortermpun ${ }^{a}$, J. W. Bieber ${ }^{c}$, A. C.-L. \\ Chian $^{d, e, f}$ \\ ${ }^{a}$ Department of Physics, Faculty of Science, Mahidol University, Bangkok 10400, Thailand. \\ ${ }^{b}$ Department of Physics, Faculty of Science, Benue State University, Makurdi PMB 102119, \\ Nigeria. \\ ${ }^{c}$ Bartol Research Institute, University of Delaware, Newark, DE 19716, USA. \\ ${ }^{d}$ School of Mathematical Sciences, University of Adelaide, Adelaide, SA 5005, Australia. \\ eInstitute of Aeronautical Technology (ITA), São José dos Campos, SP, 12228-900, Brazil. \\ ${ }^{e}$ National Institute for Space Research (INPE), P.O. Box 515, São José dos Campos, SP, \\ 12227-010, Brazil. \\ E-mail: domgrace1@gmail.com, david.ruf@mahidol.ac.th.
}

The flux of Galactic cosmic rays (GCRs) can undergo a Forbush decrease (FD) during the passage of a shock, sheath region, or magnetic flux rope associated with a coronal mass ejection (CME). Cosmic-ray observations during FDs can provide information complementary to in situ observations of the local plasma and magnetic field, because cosmic-ray distributions allow remote sensing of distant conditions. Here we develop techniques to determine the GCR anisotropy before and during an FD using data from neutron monitors worldwide. For example, for the FD starting on 2013 April 13, we find that at times with strong magnetic fluctuations and strong cosmic-ray scattering, there were spikes of high perpendicular anisotropy and weak parallel anisotropy. These results, along with the near constancy of parallel anisotropy across magnetic field reversals, are consistent with diffusive barriers causing the decrease in GCR flux before the arrival of the flux rope. In contrast, within the CME flux rope there was a strong parallel anisotropy in the direction predicted from a theory of drift motions into one leg of the magnetic flux rope and out the other, confirming that the anisotropy can remotely sense a large-scale flow of GCRs through a magnetic flux rope.

36th International Cosmic Ray Conference -ICRC2019-

July 24th - August 1st, 2019

Madison, WI, U.S.A.

${ }^{*}$ Speaker. 


\section{Introduction}

The flux of GCRs can undergo a FD during the passage of a shock, sheath region or magnetic flux rope associated with a CME [1,2], which can also cause variations in the distribution of GCR.

Consequently, the significance of GCR anisotropy and FD cannot be overemphasized. For instance, it can provide very important and useful information on the properties of solar wind plasma $[1,2,3,4,5,6]$. In addition to the above, a good knowledge of GCR anisotropy can provide remote information about the structure of CME flux ropes, and this could be related to various space weather effects and space weather forecasting. GCR anisotropy can also shed light on understanding the propagation of GCR [7].

With the aforementioned motivation, there have been comprehensive articles about the characteristics of Forbush decreases and their causes, where the arrival of a CME shock has been identified as the major cause of Forbush decrease [1, 8, 9, 10, 11].

The anisotropy of GCRs has also been investigated by several authors. For example, CMEs can cause depressions in the cosmic ray intensity both locally, when an observer is inside the interplanetary structure (ejecta) and remotely, if the ejecta is energetic enough to create an interplanetary shock to which the observer is magnetically connected. After the shock and ejecta have passed, the intensity gradually recovers as particles diffuse in around the shock.

Following the previous studies, we have modeled GCR anisotropy using a technique initially developed by [2], alongside normalized count rates from NM stations. In this analysis, we improve the technique by using only polar stations to make the process cleaner and we have also calculated the actual asymptotic directions for each hour so the actual asymptotic directions calculated using the approximate $K_{p}$ indices are used for each hour in the analysis. This is in contrast to the previous procedure where the asymptotic directions calculated for all hours of one day were used across all days throughout the analysis. Another important improvement of the technique is that we have redefined the daily average.

For the FD starting on 2013 April 13, we find that at times with strong magnetic fluctuations and strong cosmic-ray scattering, there were spikes of high perpendicular anisotropy and weak parallel anisotropy. These results, along with the near constancy of parallel anisotropy across magnetic field reversals, are consistent with diffusive barriers causing the decrease in GCR flux before the arrival of the flux rope. Within the CME flux rope there was a strong parallel anisotropy in the direction predicted from a theory of drift motions into one leg of the magnetic flux rope and out the other [22], confirming that the anisotropy can remotely sense a large-scale flow of GCRs through a magnetic flux rope.

\section{Data Information and Procedure}

\subsection{Data Information}

We used data from polar NM stations with good statistics and no major data gaps during the period of FD. These stations and their geographic locations along with their geomagnetic cutoff rigidity are presented in table 1 . 
The neutron monitor data were obtained from the Neutron Monitor Database ${ }^{1}$. We used data from all available polar stations in this database of the worldwide network of neutron monitors with good data for the time period considered. We also used level 2 data from the ACE spacecraft ${ }^{2}$ for the solar wind speed, magnitude (B) and geocentric solar-ecliptic (GSE) components of the interplanetary magnetic field.

Table 1: Sources of Neutron Monitor Data

\begin{tabular}{lcccc}
\hline \hline NM Location & $\begin{array}{c}\text { Station } \\
\text { Code }\end{array}$ & $\begin{array}{c}\text { Geographic } \\
\text { Latitude } \\
(\mathrm{deg})\end{array}$ & $\begin{array}{c}\text { Geographic } \\
\text { Longitude } \\
P_{c}(\mathrm{GV})\end{array}$ & $\begin{array}{c}\text { Cutoff } \\
\text { Rigidity } \\
P_{m}(\mathrm{GV})\end{array}$ \\
\hline Thule, Greenland & TH & 76.50 & -68.70 & 0.00 \\
Fort Smith, Canada & FS & 60.02 & -111.93 & 0.00 \\
Peawanuck, Canada & PE & 54.98 & -85.44 & 0.00 \\
Barentsburg, Russia & BA & 78.06 & 14.21 & 0.00 \\
McMurdo, Antarctica & MC & -77.90 & 166.60 & 0.00 \\
Terre Adélie, Antarctica & TA & -66.55 & 140.00 & 0.00 \\
Nain, Canada & NA & 56.55 & -61.68 & 0.01 \\
South Pole, Antarctica & SP & -90.00 & 0.00 & 0.09 \\
Inuvik, Canada & IN & 68.36 & -133.72 & 0.18 \\
Mawson, Antarctica & MA & -67.60 & 62.87 & 0.22 \\
Jang Bogo, Antarctica & JB & -74.62 & 164.23 & 0.30 \\
Tixie Bay, Russia & TB & 71.36 & 128.54 & 0.48 \\
Norilsk, Russia & NO & 69.26 & 88.05 & 0.58 \\
Apatity, Russia & AP & 67.57 & 33.40 & 0.65 \\
Oulu, Finland & OU & 65.05 & 25.47 & 0.81 \\
SANAE, Antarctica & SA & -70.32 & -2.35 & 1.06 \\
Kerguelen, near Antarctica & KE & -49.35 & 70.25 & 1.14 \\
\hline
\end{tabular}

\subsection{Procedure}

The first order anisotropy $\boldsymbol{\delta}$ of galactic cosmic rays can be related to the count rate of NM station $n$ at a given time $t$, using the following general equation [2]:

$$
\begin{aligned}
A_{n}(t) & =\sum_{l=0}^{8} w_{l} \int_{P_{\min , n, l}}^{P_{\max }}\left(\left.\frac{-d N\left(P_{c}\right)}{d P_{c}}\right|_{P}\right) T_{n, l}(P) \frac{P}{D(t)+P} \\
& \times\left\{1+\left[\delta_{x}(t)+\chi_{x}(t, P)\right] n_{n, l, x}(t, P)+\left[\delta_{y}(t)+\chi_{y}(t, P)\right] n_{n, l, y}(t, P)\right. \\
& \left.+\left[\delta_{z}(t)+\chi_{z}(t, P)\right] n_{n, l, z}(t, P)\right\} d P .
\end{aligned}
$$

\footnotetext{
${ }^{1}$ htp://www.nmdb.eu

${ }^{2}$ htp://http://www.srl.caltech.edu/ACE/ASC
} 
In equation $2.1, l$ is one of 9 directions with weights $w_{l}$ set to $1 / 2$ for the vertical direction and $1 / 16$ for the other 8 directions at zenith angle $30^{\circ}$ and azimuthal angles equally spaced over $22^{\circ} .5-337^{\circ} .5[2,13] . N\left(P_{c}\right)$ is the NM response function, and the Dorman fit from a latitude survey during $2004-2005$ was used for this $[2,14]$. This survey year was selected because it is the survey year for which the modulation of galactic cosmic rays was similar to that during 2013. The transmission function $T_{n l}(P)$ is set to 1 in this analysis because only polar stations were used. $\delta_{x}, \delta_{y}$ and $\delta_{z}$ represent the first order anisotropy in $G E O_{x}, G E O_{y}$, and $G E O_{z}$-components respectively, and $\boldsymbol{\chi}$ is the Compton-Getting anisotropy. Finally, the unit vector $\boldsymbol{n}(t, P)$ accounts for the asymptotic directions of GCR at NM stations; to find the asymptotic directions we used a particle trajectory code from the Bartol Research Institute $[15,16]$ together with an accurate model of the terrestrial magnetic field including any field disturbance present as indicated by the $K_{p}$ index value at each hour.

To avoid a mis-representation of the fixed North-South anisotropy $\left(\delta_{z}(t)\right)$ and equatorial anisotropy $\left(\delta_{x}(t)\right.$ and $\left.\delta_{y}(t)\right)$, we first evaluate $\delta_{z}(t)$ in GEO coordinates using the following equation [2]:

$$
\frac{T(t)-M(t)}{2 P_{t}(t)}=\frac{\sum_{l=0}^{8} w_{l} \int_{P_{\min , n, l}}^{P_{\max }}\left(\left.\frac{-d N\left(P_{c}\right)}{d P_{c}}\right|_{P}\right) T_{n, l}(P) \frac{P}{D(t)+P}\left[\delta_{z}(t)+\chi_{z}(t, P)\right]\left[n_{T, l, z}(t, P)-n_{M, l, z}(t, P)\right] d P}{2 \sum_{l=0}^{8} w_{l} \int_{P_{\min }}^{P_{\max }}\left(\left.\frac{-d N\left(P_{c}\right)}{d P_{c}}\right|_{P}\right) d P}
$$

where $T(t)$ and $M(t)$ are the normalized count rates of Thule and McMurdo, respectively. $P_{t}(t)$ is the average count rate of all polar stations. Here, we have used only polar stations with atmospheric cutoff rigidity approximately $1 \mathrm{GV}$ and as such the transmission $T=1$.

Then we determine the equatorial anisotropy $\left(\delta_{x}(t)\right.$ and $\left.\delta_{y}(t)\right)$ by performing a least squares fit to equation (2.3) for all NM stations simultaneously:

$$
\frac{C_{n}(t)}{C_{n, d}(t)}=\frac{A_{n}(t)}{B_{n}(t)}
$$

From (2.3), $C_{n}(t)$ is the count rate of individual stations at a given time $t, A_{n}(t)$ represents the modeled count rate, $B_{n}(t)$ models the daily average over equatorial directions and is equal to $A_{n}(t)$ but with $n_{n, l, x}=n_{n, l, y}=0$, and $C_{n, d}(t)$ is the daily running average defined by equation (2.4). We calculate this by defining $C_{n}^{\prime}(t)$ as the NM count rate $C_{n}(t)$ divided by the average count rate $\left(P_{t}(t)\right)$. Then we use equation (2.4) and multiply the result by $P_{t}(t)$ :

$$
C_{n, d}^{\prime}(t)=\frac{1}{2}\left[C_{n}^{\prime}(t)+\left(\frac{C_{n}^{\prime-}(t)+C_{n}^{\prime+}(t)}{2}\right)-\frac{1}{4}\left(\frac{C_{n}^{\prime--}(t)+C_{n}^{\prime++}(t)}{2}-C_{n}^{\prime}(t)\right)\right],
$$

where $C_{n}^{\prime-}(t), C_{n}^{\prime--}(t), C_{n}^{\prime+}(t)$ and $C_{n}^{\prime++}(t)$ are the count rate ratios of same NM station at times $t-12$ hours, $t-24$ hours, $t+12$ hours and $t+24$ hours respectively. Then we use $C_{n, d}(t)=$ $C_{n, d}^{\prime}(t) P_{t}(t)$. The daily running average as defined in equation (2.4) is designed to average over 
any daily wave in the normalized count rates. Then $C_{n, d}(t)$ is modeled using $B_{n}(t)$. Examples of $C_{n}(t) / C_{n, d}(t)$ are given in Figure 1



Figure 1: Count rates divided by daily running average for the FD starting 2013 April 13 for selected NM stations. Such ratios for each station are considered to deviate from 1 due to equatorial anisotropy as expressed by Eq. 2.3

The anisotropies obtained from the procedure described in section 2 are first converted from GEO coordinates to GSE coordinates and then further decomposed into components parallel and perpendicular to the interplanetary magnetic field $\boldsymbol{B}$. This is to enable us interpret the anisotropy results in the solar wind frame.

\section{Results and Discussion}

In order to accurately interpret our results, we first analyze plasma and magnetic field data to identify the distinct structures in the interplanetary coronal mass ejections (ICMEs), as well as shock arrival times and possible flux ropes, alongside hourly count rates from NM stations. Figure 2a shows plasma data and magnetic field parameters from the Wind spacecraft. Figure $2 b$ shows hourly count rates from selected NM stations before and during a FD. Figure $2 \mathrm{c}$ shows our results for first order cosmic-ray anisotropy ${ }^{3}$. Figure $2 \mathrm{~d}$ shows the cosmic-ray anisotropy magnitude in the solar wind frame perpendicular and parallel to the interplanetary magnetic field from the

\footnotetext{
${ }^{3}$ Note that $\delta_{x}$ was misplotted in [2]
} 
ACE spacecraft. Here $B, B_{z}, \Delta B_{r m s}, T_{p}, V_{p}, \theta, \phi$, sym- $\mathrm{H}$, and $\left|V_{s w}\right|$ are magnetic field magnitude, $z$-component of magnetic field, root mean square fluctuation of magnetic field magnitude, proton temperature, proton flow speed, latitude of magnetic field vector, longitude of magnetic field vector, longitudinally symmetric disturbance index for the horizontal (dipole) direction and the modulus of solar wind speed respectively. Then $S, B 1, B 2$, and MC stand for the shock arrival time, times of intense magnetic fluctuations and magnetic cloud respectively. $\delta_{x}, \delta_{y}, \delta_{z}, \delta_{\perp}$ and $\delta_{\|}$represent cosmic-ray first order anisotropy in GSE $X, Y$ and $Z$ coordinates. All times from ACE and Wind, which are located at the $L_{1}$ Lagrangian point, are shifted one hour later when comparing with data at Earth to roughly account for the solar wind travel time.

Grouping of stations in Figure $2 \mathrm{~b}$ is according to similar viewing directions. For example, dark red is for stations in Europe, dark blue is for Russian stations and light blue is for stations in Antarctica. Notations at the right of the plot indicate the station code (first letters of two words in a station name or first two letters of one-word name) and geomagnetic cut-off rigidity in GV. Note that for polar stations, the response to cosmic rays is determined by an atmospheric cutoff of $\approx 1$ GV. Arrows at the right are to show Northward and Southward asymptotic directions at Thule (TH) and McMurdo (MC) respectively.

Our results revealed an anti-correlation between $\delta_{\|}$and $\delta_{\perp}$. For example, there is often an increase in $\delta_{\perp}$ at times with stronger rms fluctuation $\Delta B_{r m s}$. This is consistent with diffusive anisotropy and theories of perpendicular diffusion $[2,23,24]$, that perpendicular diffusion increases with increasing $\left(\Delta B_{r m s} / B\right)$.

In contrast, $\delta_{\|}$was generally lower during times with stronger magnetic fluctuations and higher during times of weak fluctuations, for instance, within a CME flux rope where magnetic fluctuations are very weak. This is in good agreement with theoretical expectations that strong fluctuations can cause strong cosmic ray scattering leading to low parallel diffusion coefficient [2]. This is also consistent with the idea that a parallel diffusive barrier is responsible for the decrease of cosmic ray flux in the sheath region [19]. Note that in Figure 2d, we indicate the sign of $\boldsymbol{\delta}_{\|}$in terms of higher GCR flux when viewing along $+\boldsymbol{B}$ (blue) or $-\boldsymbol{B}$ (red) ${ }^{4}$. The color often changes with a reversal of $\boldsymbol{B}$, which is consistent with the idea that parallel scattering and flow processes do not necessarily depend on the sign of $\boldsymbol{B}$.

Within the CME flux rope there was a strong parallel anisotropy in the direction predicted from a theory of drift motions into one leg of the magnetic flux rope and out the other [22], confirming that the anisotropy can remotely sense a large-scale flow of GCRs through a magnetic flux rope. These methods have also been applied to other FD events, as will be addressed in further work.

\footnotetext{
${ }^{4}$ Colors were also incorrectly applied to $\delta_{||}$in [2]
} 
(a)

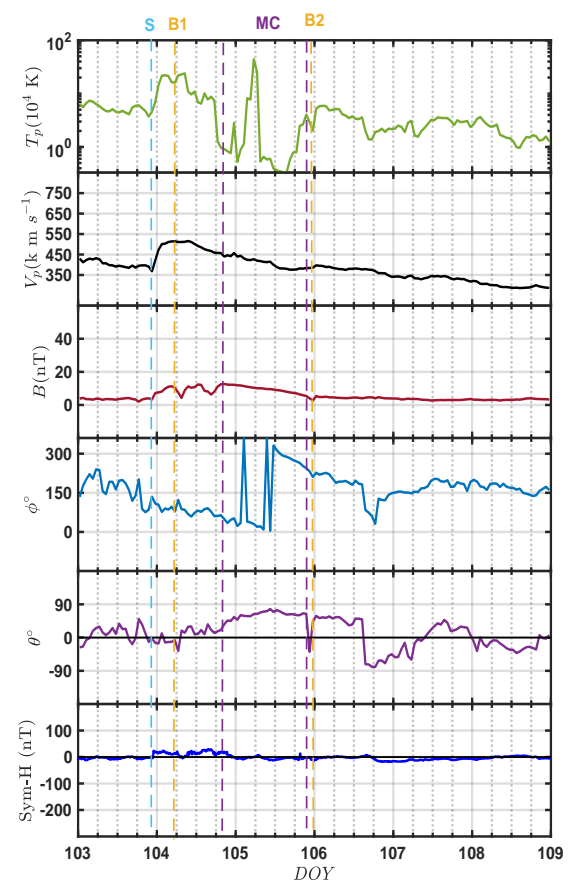

(c)

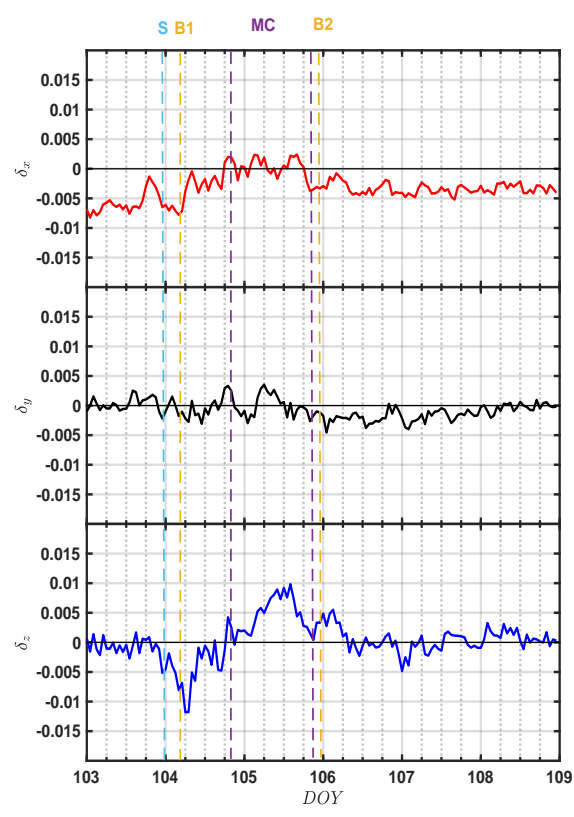

(b)

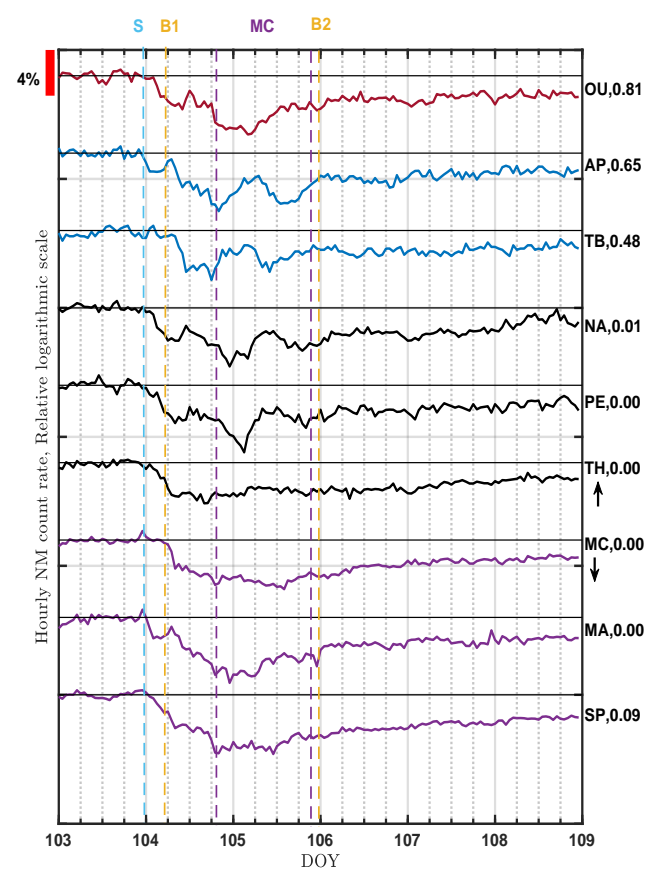

(d)

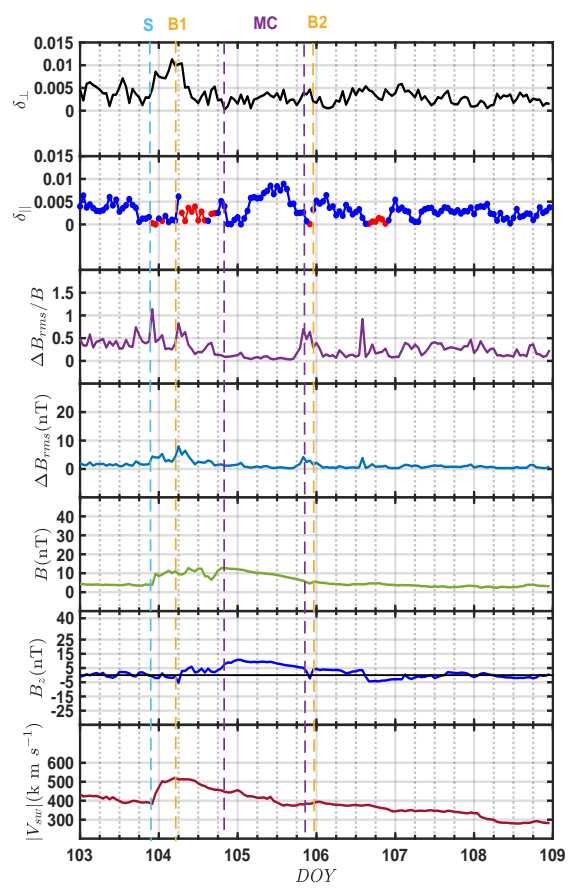

Figure 2: Results of our analysis for the FD starting 13 April 2013. See text for details. 


\section{Acknowledgments}

This study was supported by a Postdoctoral Fellowship from Mahidol University and the Thailand Research Fund via grant RTA5980003.

\section{References}

[1] Forbush, S. E. 1937, Phys. Rev, 51, 1108.

[2] Tortermpun, U., Ruffolo, D. and Bieber J. W. 2018, The Astrophysical Journal Letters, 852: L26.

[3] Belov, A. V., Bieber, J.W., Eroshenko, E.A., Evenson, P., Pyle, R., and Yanke, G.V. 2003, Adv. Space Res. Vol. 31, No 4, pp. $919-924$.

[4] Yurchyshyn V. B., Wang H., Goode, P. R. and Deng, Y. 2001, The Astrophysical Journal 563, $381-388$.

[5] Liu, Y., Manchester, W. B., IV., Kasper, J.C., Richardson, D.J. and Belcher, W.J. 2007, The Astrophysical Journal 665: 1439 - 1447.

[6] Tsurutani, B.T. and Gonzalez, W.D., 1997, in Magnetic Storms, Geophys. Monogr. Ser., Vol. 98, pp. $77-89$.

[7] Amenomori M., et al. 2017,The Astrophysical Journal $836: 153$.

[8] Cane H. V., 2000, Space Science Reviews 93:55- 77.

[9] Lockwood, J. A.: 1971, Space Sci. Revs. 12, 658-715.

[10] Iucci, N., Pinter, S., Parisi, M., Storini, M., and Villoresi, G., 1986, Nuovo Cimento 9C, 39-50.

[11] Barnden, L. R.: 1973, "Forbush Decreases 1966-1972; Their Solar and Interplanetary Associations and Their Anisotropies", Proc. 13th Int. Cosmic Ray Conf. 2, 1271-1276.

[12] Aguilar, M., Aisa, D., Alpat, B., et al. 2015, PhRvL, 114, 171103.

[13] Bieber, J. W., Clem, J., and Evenson, P. 1997, Proc. ICRC (Durban), 2, 389

[14] Nuntiyakul W., Evenson P., Ruffolo D., Sáiz, A., Bieber, J. W., Clem, J., Pyle, R., Duldig, M. L., and Humble, J. E., 2014, ApJ, 795, 11

[15] Lin Zhongmin, Bieber, J. W., Evenson P. 1995, Journal of Geophysical Research, 100, NO. A12.

[16] Nuntiyakul, W., Evenson, P., Ruffolo, D., Sáiz, A., Bieber, J. W., Clem, J., Pyle R., Duldig, M. L., and Humble, J. E., 2018, Journal of Geophysical Research: Space Physics, 123,7181.

[17] Hapgood, M.A. 1992, Planet. Space Sci., Vol 40,No5.pp.711 - 717

[18] Russell, T.C. 1971, Cosmic Electrodynamics, 2,184- 196.

[19] Wibberenz, G., le Roux, J. A., Potgieter, M. S., and Bieber, J. W. 1998, SSRv,83, 309

[20] Nagashima, K., Fujimoto, K., Sakakibara, S., Morishita, I., and Tatsuoka, R.1992, P \& SS, 40, 1109.

[21] Fushishita, A., Kuwabara, T., Kato, C., et al. 2010, ApJ, 715, 1239.

[22] Krittinatham, W., and Ruffolo, D. 2009, ApJ, 704, 831.

[23] Shalchi, A. 2010, ApJL, 720, L127.

[24] Ruffolo, D., Pianpanit, T., Matthaeus, W. H., \& Chuychai, P. 2012, ApJ, 747, L34. 